\title{
Evidence For Hmgn2 Involvement in Mouse Embryo Implantation and Decidualization
}

\author{
Dang-Dang Lia Liang Yue ${ }^{b} \quad$ Zhan-Qing Yang ${ }^{a} \quad$ Lian-Wen Zheng $^{c}$ Bin Guo \\ aCollege of Veterinary Medicine, Jilin University, Changchun, ${ }^{\mathrm{b} C o l l e g e}$ of Clinical Medicine, Jilin \\ University, Changchun, 'Reproductive Medical Center, the Second Hospital of Jilin University, \\ Changchun, P. R. China
}

\section{Key Words}

Hmgn2 • Embryo implantation • Decidualization • Uterus

\begin{abstract}
Background/Aims: Hmgn2 is involved in regulating embryonic development, but its physiological function during embryo implantation and decidualization remains unknown. Methods: In situ hybridization, real-time PCR, RNA interference, gene overexpression and MTS assay were used to examine the expression of Hmgn2 in mouse uterus during the preimplantation period and explore its function and regulatory mechanisms in epithelial adhesion junction and stromal cell proliferation and differentiation. Results: Hmgn2 was primarily accumulated in uterine luminal epithelia on day 4 of pregnancy and subluminal stromal cells around the implanting blastocyst at implantation sites on day 5. Similar results were observed during delayed implantation and activation. Meanwhile, Hmgn2 expression was visualized in the decidua. In uterine epithelial cells, silencing of Hmgn2 by specific siRNA reduced the expression of adhesion molecules Cdh1, Cdh2 and Ctnnb1 and enhanced the expression of Muc1, whereas constitutive activation of Hmgn2 exhibited the opposite effects, suggesting a role for Hmgn2 in attachment reaction during embryo implantation. Estrogen stimulated the expression of Hmgn2 in uterine epithelia, but the stimulation was abrogated by ER antagonist ICI 182,780. Further analysis evidenced that attenuation of Hmgn2 might eliminate the regulation of estrogen on the expression of $\mathrm{Cdh} 1, \mathrm{Cdh} 2$ and Ctnnb1. In uterine stromal cells, progesterone induced the accumulation of Hmgn2 which advanced the expression of Prl8a2 and $\operatorname{Pr} 3 \mathrm{c} 1$, two well-known differentiation markers for decidualization, but did not affect the proliferation of stromal cells. Knockdown of Hmgn2 blocked the progesterone-induced differentiation of uterine stromal cells. Moreover, Hmgn2 might serve as an intermediate to mediate the regulation of progesterone on Hand2. Conclusion: Hmgn2 may play an important role during embryo implantation and decidualization.
\end{abstract}

D. Li and L. Yue contributed equally to this work.

Lian-Wen Zheng

and Bin Guo
College of Veterinary Medicine, Jilin University, Changchun, (P. R. China)

E-Mail362683485@qq.com, guobin79@jlu.edu.cn

\section{KARGER}




\section{Introduction}

Successful implantation, which requires synchronization between an implantationcompetent blastocyst and a receptive uterus, is critical for the establishment of pregnancy [1-4]. In humans, $15 \%$ of couples worldwide are childless due to infertility, the major cause of which is considered to be implantation failure $[1,5,6]$. Accompanying the initiation of embryo implantation, uterine stromal cells undergo extensive proliferation and then differentiation into decidual cells, a process known as decidualization, which may nourish the developing embryo and protect it from the maternal rejection before placenta maturation [2, 7]. Inadequate decidualization can result in recurrent miscarriage, unexplained infertility or even pathological pregnancy $[7,8]$. Accumulated data have evidenced that ovarian estrogen and progesterone are master regulators of embryo implantation and decidualization $[1,2]$, but their underlying regulatory mechanisms remain largely unknown.

High mobility group nucleosomal binding domain 2 (Hmgn2), also referred to as high mobility group protein 17 (Hmg17), is an abundant member of the non-histone Hmgn family that can specifically bind to nucleosome core particle, alter chromatin structure and affect DNA-dependent activities such as transcription, replication and repair $[9,10]$. Previous study has found that Hmgn2 was present in early embryos, peaking in the blastocyst, and its deficiency led to embryonic lethality $[9,11,12]$. Moreover, transient depletion of Hmgn $1 / 2$ delayed the progression of preimplantation embryonic development [12]. However, the biological function of Hmgn2 in embryo implantation is still unknown. Meanwhile, Hmgn2 might promote astrocyte differentiation of neural precursor cells and antagonize erythroid differentiation $[13,14]$. According to our (unpublished) microarray data, Hmgn2 was strongly expressed in day 8 decidua and deciduoma under artificial decidualization compared with the uninjected uterine horn, but it is unclear whether Hmgn2 may regulate the differentiation of uterine stromal cells in response to progesterone.

In this study, we showed that Hmgn2 might play an important role in embryo implantation and decidualization, and act downstream of progesterone to regulate the differentiation of uterine stromal cells through targeting Hand2.

\section{Materials and Methods}

\section{Animals}

Mature mice (Kunming white strain) were caged in a controlled environment with a cycle of 14L:10D. All animal procedures were approved by the Institutional Animal Care and Use Committee of Jilin University. To confirm reproducibility of results, at least three mice per group were used in each stage or treatment in this study.

\section{Pregnancy and pseudopregnancy}

Adult female mice were mated with fertile or vasectomized males of the same strain to induce pregnancy or pseudopregnancy by co-caging, respectively (day 1=day of vaginal plug). On days 1-4, pregnancy was confirmed by recovering embryos from the oviducts or uterus. The implantation sites on day 5 were identified by intravenous injection of $0.1 \mathrm{ml}$ of $1 \%$ Chicago blue (Sigma, St. Louis, MO) in $0.85 \%$ sodium chloride.

\section{Delayed implantation and activation}

To induce delayed implantation, pregnant mice were ovariectomized under ether anesthesia at 08:3009:00h on day 4 of pregnancy. Progesterone ( $1 \mathrm{mg} / \mathrm{mouse}$; Sigma) was injected subcutaneously to maintain delayed implantation from days 5 to 7 . Estradiol- $17 \beta$ (25 ng/mouse, Sigma) was given to progesteroneprimed delayed-implantation mice to activate blastocyst implantation. The mice were sacrificed to collect uteri $24 \mathrm{~h}$ after estrogen treatment. The implantation sites were identified by intravenous injection of Chicago blue solution. Delayed implantation was confirmed by flushing the blastocysts from the uterus. 


\section{Cellular Physiology Cell Physiol Biochem 2017;44:1681-1695 \begin{tabular}{ll|l} 
and Biochemistry Published onlIne: December 06, 2017 & $\begin{array}{l}\text { (c) } 2017 \text { The Author(s). Published by S. Karger AG, Basel } \\
\text { www.karger.com/cpb }\end{array}$
\end{tabular}}

Li et al.: Hmgn2 Regulation in Mouse Uterus

\section{Artificial induced decidualization}

Artificial decidualization was induced by intraluminally infusing $25 \mu$ of sesame oil into one uterine horn on day 4 of pseudopregnancy, while the contralateral uninjected horn served as a control. The mice were killed to collect uteri at $24,48,72$ or $96 \mathrm{~h}$ after artificial induced decidualization. Decidualization was confirmed by weighing the uterine horn and histological examination of uterine sections.

\section{Steroid hormonal treatments}

Mature female mice were ovariectomized and, after 2 weeks, given a single injection of estradiol-17 $\beta$ (100 ng/mouse), progesterone ( $2 \mathrm{mg} / \mathrm{mouse}$ ) or a combination of the same doses of estradiol- $17 \beta$ and progesterone, respectively. All steroids were dissolved in sesame oil and injected subcutaneously. Control mice received the vehicle only (sesame oil, $0.1 \mathrm{ml} /$ mouse). Mice were sacrificed at different times to collect uteri after hormonal injections.

Uterine epithelial and stromal cells from day 4 of pregnancy were isolated and cultured as previously described [15]. Cultured epithelial and stromal cells were also treated with $1 \mu \mathrm{M}$ of progesterone or $0.1 \mathrm{nM}$ of estradiol-17 $\beta$, respectively. For further studies, cells were pretreated with RU486 $(1 \mu \mathrm{M})$ for $2 \mathrm{~h}$ or ICI $182,780(100 \mathrm{nM})$ before the addition of progesterone or estrogen, respectively. Steroids and antagonists were dissolved in ethanol. Controls received the vehicle only.

\section{In situ hybridization}

Total RNAs from the mouse uteri were reverse-transcribed and amplified with Hmgn2 primers. Hmgn2 forward primer 5'- TCCCAGCGCTATAAAAACT and reverse primer 5'- TGGTTTTGTCTCCTTTAGCA were designed according to Mus musculus high mobility group nucleosomal binding domain 2 gene (Genbank accession number NM_016957). The amplified fragment (182 bp) of Hmgn2 was cloned into pGEM-T plasmid (pGEM-T Vector System 1, Promega, Madison, WI) and verified by sequencing. Hmgn2-containing plasmid was amplified with the primers for T7 and SP6 to prepare templates for labeling. Digoxigenin (DIG)labeled antisense and sense cRNA probes were transcribed in vitro using a DIG RNA labeling kit (Roche Diagnostics GmbH, Mannheim, Germany).

Frozen sections $(10 \mu \mathrm{m})$ were mounted on 3-aminopropyltriethoxy-silane (Sigma)-coated slides and fixed in 4\% paraformaldehyde solution in PBS. Hybridization was performed as described previously [15]. Sections were counterstained with $1 \%$ methyl green. The positive signal was visualized as a dark brown color. The sense probe was also hybridized and served as a negative control. There was no detectable signal from sense probes.

Real-time PCR

Total RNAs from mouse uteri or cultured cells were isolated using TRIPURE reagent according to the manufacturer's instructions (Roche) and reverse-transcribed into cDNA using M-MLV reversetranscriptase (Promega). Reverse transcription was performed at $42^{\circ} \mathrm{C}$ for $60 \mathrm{~min}$ with $2 \mu \mathrm{g}$ total RNA in $25 \mu \mathrm{l}$ volume. For real-time PCR, cDNA was amplified using FS Universal SYBR Green Real Master (Roche) on BIO-RAD CFX96 ${ }^{\mathrm{TM}}$ Real Time Detection System. The conditions used for real-time PCR were as follows: $95^{\circ} \mathrm{C}$ for 3

Table 1. Primers for real-time PCR

\begin{tabular}{|c|c|c|c|}
\hline Gene & Primer sequence & Accession number & $\begin{array}{l}\text { Size } \\
\text { (bp) }\end{array}$ \\
\hline \multirow{3}{*}{ Hmgn 2} & AAAAGGCCCCTGCGAAGAA & & \\
\hline & TGCCTGGTCTGTTTTGGCA & NM_016957 & 116 \\
\hline & AACCCAAGCACGTATCAGGG & & \\
\hline \multirow[t]{2}{*}{ Cdh 1} & ACTGCTGGTCAGGATCGTTG & NM_009864 & 142 \\
\hline & GCGCAGTCTTACCGAAGGAT & & \\
\hline \multirow[t]{2}{*}{ Cdh 2} & CGTCCTCGTCCACCTTGAAA & NM_007664 & 129 \\
\hline & ACTTGCCACACGTGCAATTC & & \\
\hline \multirow[t]{2}{*}{ Ctnnb1 } & ATGGTGCGTACAATGGCAGA & NM_007614 & 165 \\
\hline & GATGCAGTTCCCTCCCTCTG & & \\
\hline \multirow[t]{2}{*}{ Mucl } & CCAGCTGCCCATAGCTCTTT & NM_013605 & 155 \\
\hline & AGCCAGAAATCACTGCCACT & & \\
\hline \multirow[t]{2}{*}{ Prlsa2 } & TGATCCATGCACCCATAAAA & NM_010088 & 119 \\
\hline & GCCACACGATATGACCGGAA & & \\
\hline \multirow[t]{2}{*}{ Prl3cl } & GGTTTGGCACATCTTGGTGTT & NM_001163218 & 162 \\
\hline & CACAGAACAAGGCCAAAGGC & & \\
\hline \multirow[t]{2}{*}{ Hand 2} & CTTGTCGTTGCTGCTCACTG & NM_010402 & 161 \\
\hline & GCCTTCCGTGTTCCTACCC & & \\
\hline Gapdh & TGCCTGCTTCACCACCTTC & NM_008084 & 102 \\
\hline
\end{tabular}




\section{Cellular Physiology Cell Physiol Biochem 2017;4:1681-1695

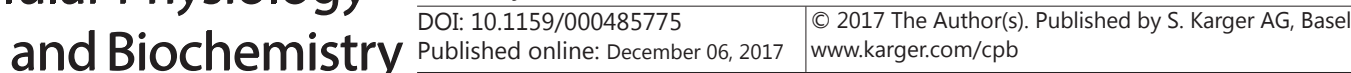

Li et al.: Hmgn2 Regulation in Mouse Uterus

min, followed by 40 cycles of $95^{\circ} \mathrm{C}$ for $15 \mathrm{~s}$ and $60^{\circ} \mathrm{C}$ for $1 \mathrm{~min}$. All reactions were run in triplicate. Results were analyzed using CFX Manager Software. After analysis using the $2^{-\Delta \Delta \mathrm{Ct}}$ method, data were normalized to Gapdh expression. Primer sequences for real-time PCR were listed in Table 1.

\section{RNA interference}

The small-interfering RNA (siRNA) duplexes for targeting Hmgn2 as well as a scrambled sequence (control siRNA duplex, negative control) were designed and synthesized by GenePharma. The sequences were shown as follows: 5'- CCUGUACUAUCAACAUAGATT and 5'- UCUAUGUUGAUAGUACAGGTT (Hmgn2 siRNA); 5'- UUCUCCGAACGUGUCACGUTT and 5'- ACGUGACACGUUCGGAGAATT (nonspecific scrambled siRNA, negative control). Transfections for siRNA were performed with Lipofectamine 2000 (Invitrogen, Carlsbad, CA) according to the manufacturer's protocol. After transfection with Hmgn2 siRNA, uterine epithelial and stromal cells were collected in the absence or presence of estrogen and progesterone, respectively.

Plasmid Construction and Transfection

Full-length Hmgn2 cDNA fragments were amplified by PCR from mouse uterus using the following primers: 5'- GAATTC (EcoRI) GCCACCATGCCCAAAAGAAAGG and 5'- GATATC (EcoRV) TCACCAGAAGTACACAGTTATC. The amplified products were purified and cloned into pGEM-T vector. The pGEM-T-Hmgn2 and pcDNA3.1 vector were cut by EcoRI/EcoRV (TaKaRa, Dalian, China) at $37^{\circ} \mathrm{C}$ for $1 \mathrm{~h}$, and then the fragments were ligated into pcDNA3.1 with T4 ligase (Promega) at $4{ }^{\circ} \mathrm{C}$ overnight to construct pcDNA3.1-Hmgn2. An empty pcDNA3.1 expression vector was served as control.

Transfection of uterine epithelial and stromal cells was performed according to the manufacturer's protocol for Lipofectamine 2000 (Invitrogen). After transfection with control plasmid (empty pcDNA3.1 vector) or Hmgn2 overexpression plasmid, epithelial and stromal cells were collected in the absence or presence of estrogen and progesterone, respectively.

\section{Cell proliferation}

Proliferation assays were performed using MTS reagent (Promega) according to the manufacturer's directions. Uterine stromal cells were seeded at a density of $1 \times 10^{5} /$ well in 96 -well plates and cultured in DMEM/F12 medium containing 2\% heat-inactivated FBS. After transfection with Hmgn2 siRNA or overexpression plasmid, stromal cells were cultured for $48 \mathrm{~h}$, at which time $20 \mu \mathrm{l}$ of MTS reagent was added to each well and incubated for $4 \mathrm{~h}$. Absorbance was measured at $490 \mathrm{~nm}$ using a 96-well plate reader. Each experiment was performed in triplicate.

\section{Statistics}

All the experiments were independently repeated at least 3 times. The significance of difference was analyzed by one-way ANOVA or Independent-Samples T Test using the SPSS software program (SPSS Inc., Chicago). The differences were considered significant at $\mathrm{P}<0.05$.

\section{Results}

\section{Hmgn2 mRNA expression during early pregnancy}

To address the physiological relevance of Hmgn2 in early pregnancy events, in situ hybridization was used to localize the distribution of Hmgn2 in mouse uterus. The results showed that Hmgn2 mRNA signal was weakly detected in the luminal epithelia on day 1 of pregnancy and its expression was visualized in the luminal and glandular epithelia from days 2 to 4 of pregnancy (Fig. 1A-D). Meanwhile, uterine stromal cells on days 3 and 4 of pregnancy also exhibited an obvious signal for Hmgn2 mRNA (Fig. 1C and D). With the onset of implantation on day 5, Hmgn2 mRNA was distinctly observed in the subluminal stromal cells around the implanting blastocyst at implantation sites as well as in the luminal and glandular epithelia, whereas there was a low level of signal at inter-implantation sites (Fig. $1 \mathrm{E}$ and $\mathrm{F}$ ). When pregnancy proceeded to days 6-8, a high level of Hmgn2 mRNA signal was consistently seen in the decidua and embryo (Fig. 1G-I). 


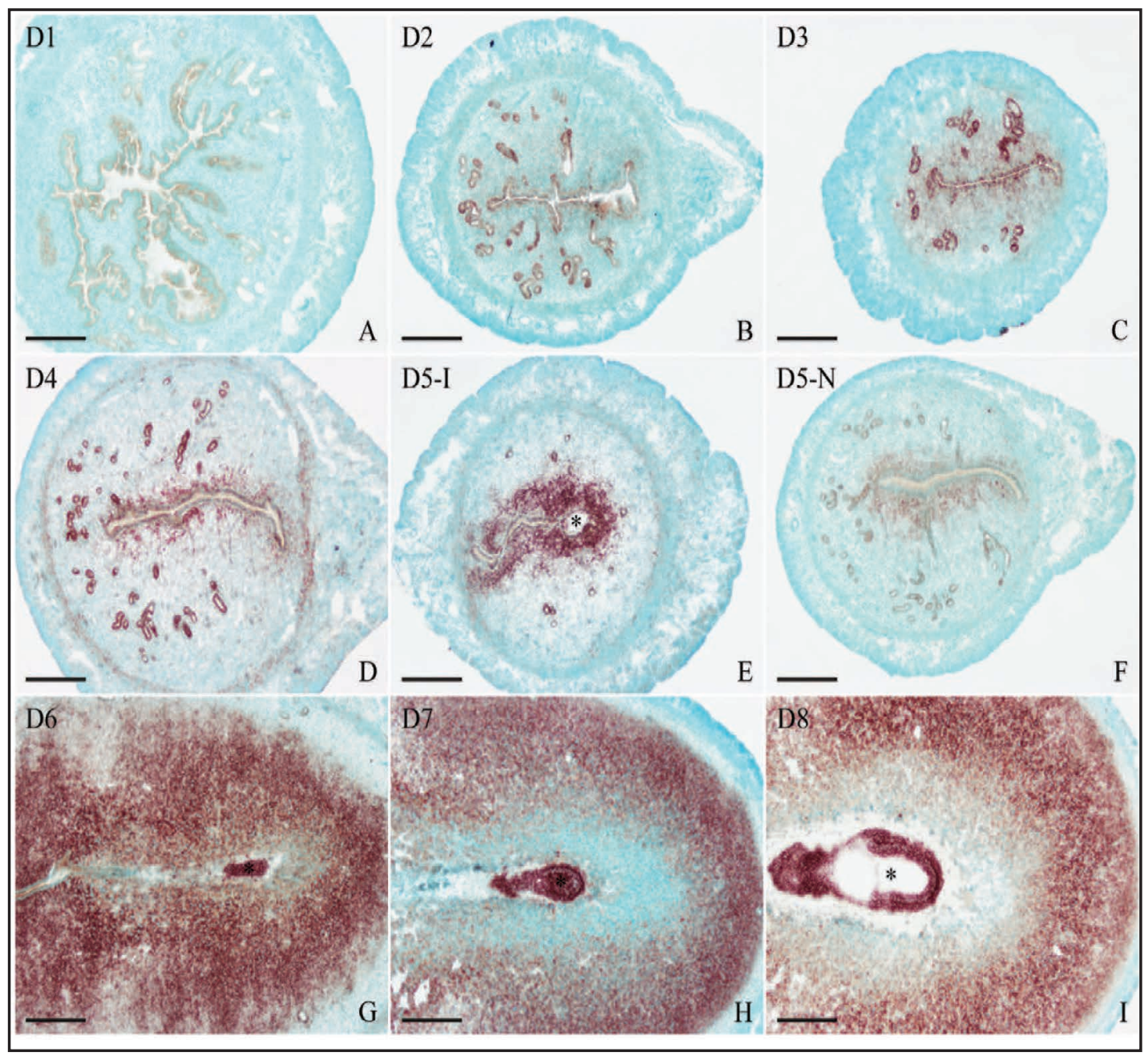

Fig. 1. In situ hybridization of Hmgn2 expression in mouse uteri during early pregnancy on days 1 (A), 2 (B), 3 (C), 4 (D), 5 (E, F), 6 (G), 7 (H), and 8 (I). 5-I, implantation site on day 5 of pregnancy; 5-N interimplantation site on day 5 of pregnancy. Asterisks indicate embryo. Bar $=60 \mu \mathrm{m}$.

To quantify Hmgn2 mRNA expression, real-time PCR was performed. The result demonstrated that Hmgn2 expression was gradually increased from day 1 to 4 of pregnancy accompanied with a slight reduction on day 5 and then sustained the highest level on days 6-8 (Fig. 2A). Compared with the inter-implantation sites, elevated expression of Hmgn2 was noted at implantation sites on day 5 of pregnancy (Fig. 2B).

\section{Hmgn2 mRNA expression during delayed implantation and activation}

To determine whether the presence of an active blastocyst is necessary for the induction of Hmgn2, a delayed implantation model was employed. In the delayed uterus, Hmgn2 mRNA was evidently localized in the luminal epithelia and stromal cells, as well as found in the glandular epithelia (Fig. 3A). After delayed implantation was terminated by estrogen treatment, a high level of Hmgn2 mRNA signal was detected in the subluminal stromal cells surrounding the implanting embryo, luminal and glandular epithelia (Fig. 3B). Simultaneously, quantitative analyses of Hmgn2 by real-time PCR also revealed that Hmgn2 expression was significantly up-regulated in the activated implantation sites compared to that in the delayed uterus (Fig. 2C). 


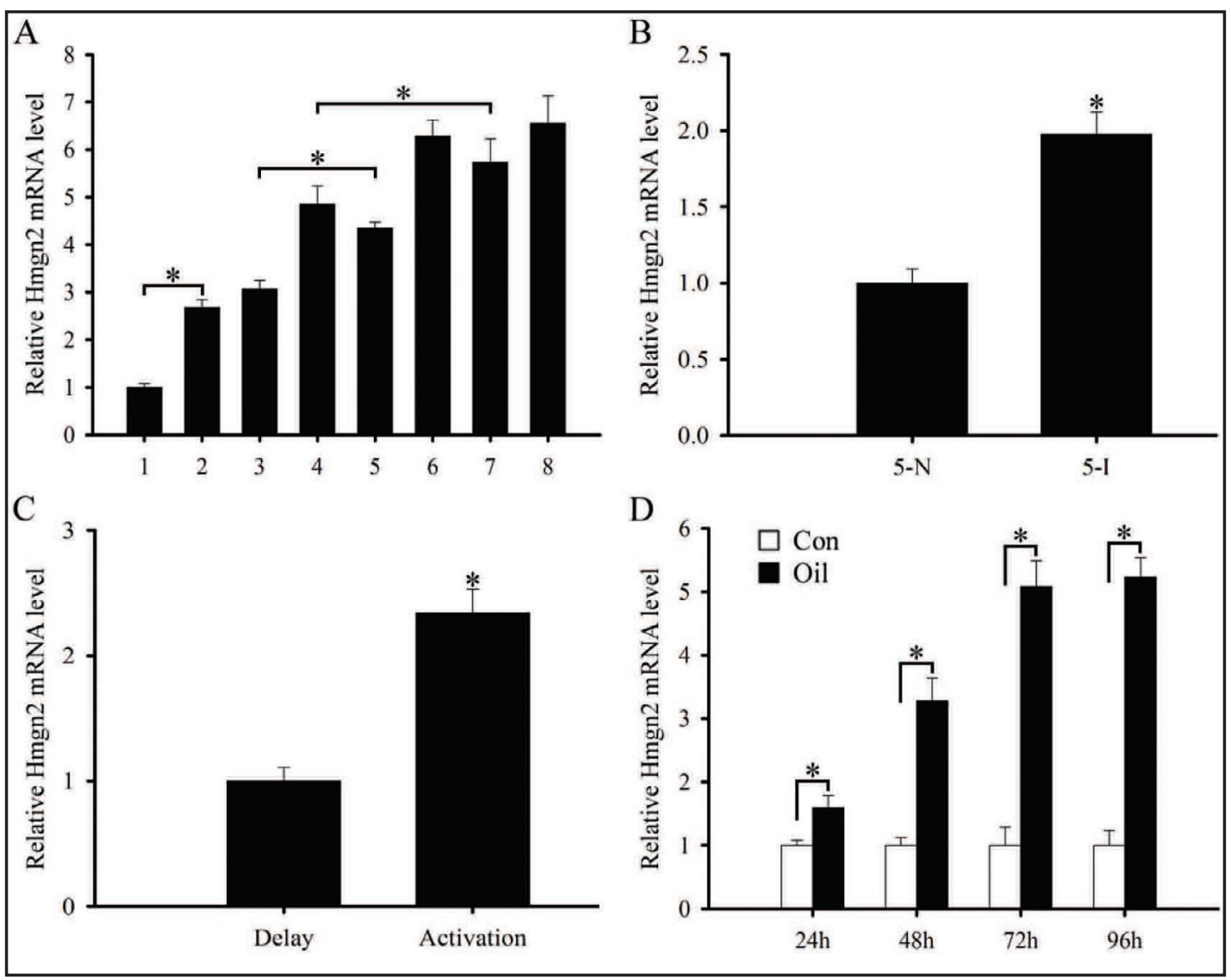

Fig. 2. Real-time PCR analysis of Hmgn2 expression in mouse uteri. A, Hmgn2 expression on days 1-8 of pregnancy. B, Hmgn2 expression at the implantation sites and inter-implantation sites on day 5 of pregnancy. C, Hmgn2 expression during delayed implantation and activation. D, Hmgn2 expression under artificial decidualization. Delay, delayed implantation; Activation, activation of delayed implantation by estrogen; Con, uninjected uterine horn; Oil, oil-induced decidualization. Data are shown mean \pm SEM. Asterisks denote significance $(\mathrm{P}<0.05)$.

\section{Hmgn2 mRNA expression under artificial decidualization}

Because Hmgn2 was highly expressed in the decidua, we also assessed its expression under artificially induced decidualization which might share many of the features of natural decidualization [16]. In the uninjected control uteri, Hmgn2 mRNA signal was barely detected at $24 \mathrm{~h}$, and primarily localized in the luminal and glandular epithelia at 48,72 and $96 \mathrm{~h}$ (Fig. 3C, E and G). After sesame oil was injected into the uterine lumen, Hmgn2 expression was visualized in the decidualizing stromal cells at different time courses (Fig. 3D, F and H). Realtime PCR analysis evidenced that after intraluminal oil infusion, the accumulation of Hmgn2 mRNA was remarkably enhanced compared with control uteri (Fig. 2D). Moreover, the enhancement was time-dependent from 24 to $72 \mathrm{~h}$, reaching a peak at $72 \mathrm{~h}$ and persisting through $96 \mathrm{~h}$ (Fig. 2D).

\section{Regulation of steroid hormones on Hmgn2 expression}

To evaluate whether ovarian steroid hormones could modulate the expression of Hmgn2, ovariectomized mice were injected with estrogen, progesterone, or estrogen plus progesterone. In the uteri of ovariectomized mice receiving sesame oil as a control, Hmgn2 mRNA signal was restricted to the luminal and glandular epithelia (Fig. 4A). After estrogen injection for $24 \mathrm{~h}$, the pattern of Hmgn2 expression was similar to that seen in control uteri but was present at much higher levels (Fig. 4B). Progesterone treatment led to an elevated 
Fig. 3. In situ hybridization of Hmgn2 expression in mouse uteri. $\mathrm{A}$ and $\mathrm{B}$, Hmgn2 expression during delayed implantation and activation. C-H, Hmgn2 expression under artificial decidualization.
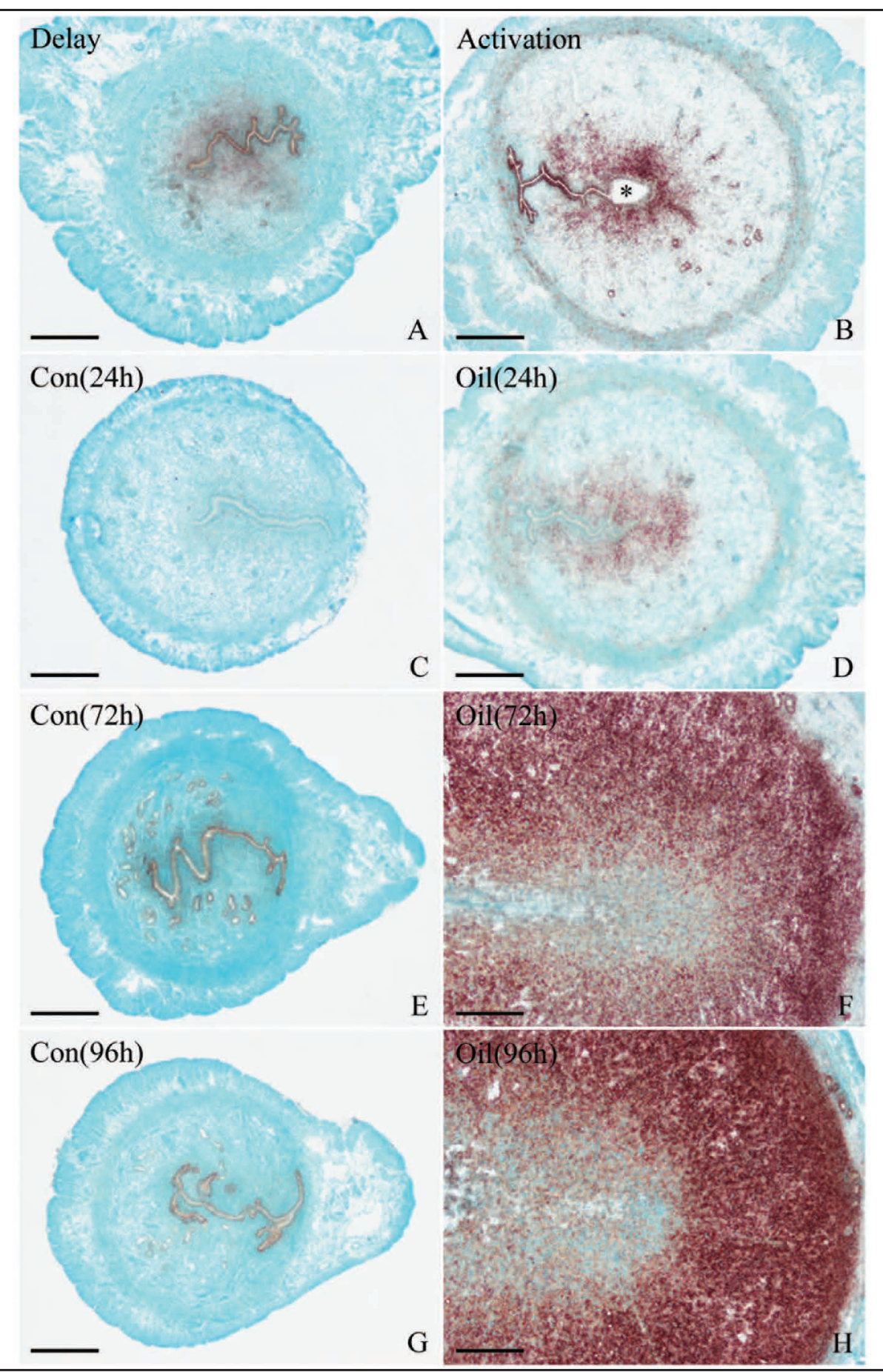

level of Hmgn2 mRNA in the luminal and glandular epithelium as well as in uterine stromal cells, which was consistent with estrogen and progesterone co-treatment (Fig. 4C and D). By real-time PCR analysis, estrogen and/or progesterone could stimulate the expression of Hmgn2 in the uteri of ovariectomized mice, but there were no any statistically significant differences between these groups (Fig. 5A).

Based on above observations, we subsequently examined the expression of Hmgn2 at different time courses after estrogen or progesterone treatment and found that Hmgn2 mRNA was visibly observed in the luminal and glandular epithelium at $3 \mathrm{~h}$ after estrogen

\section{KARGER}




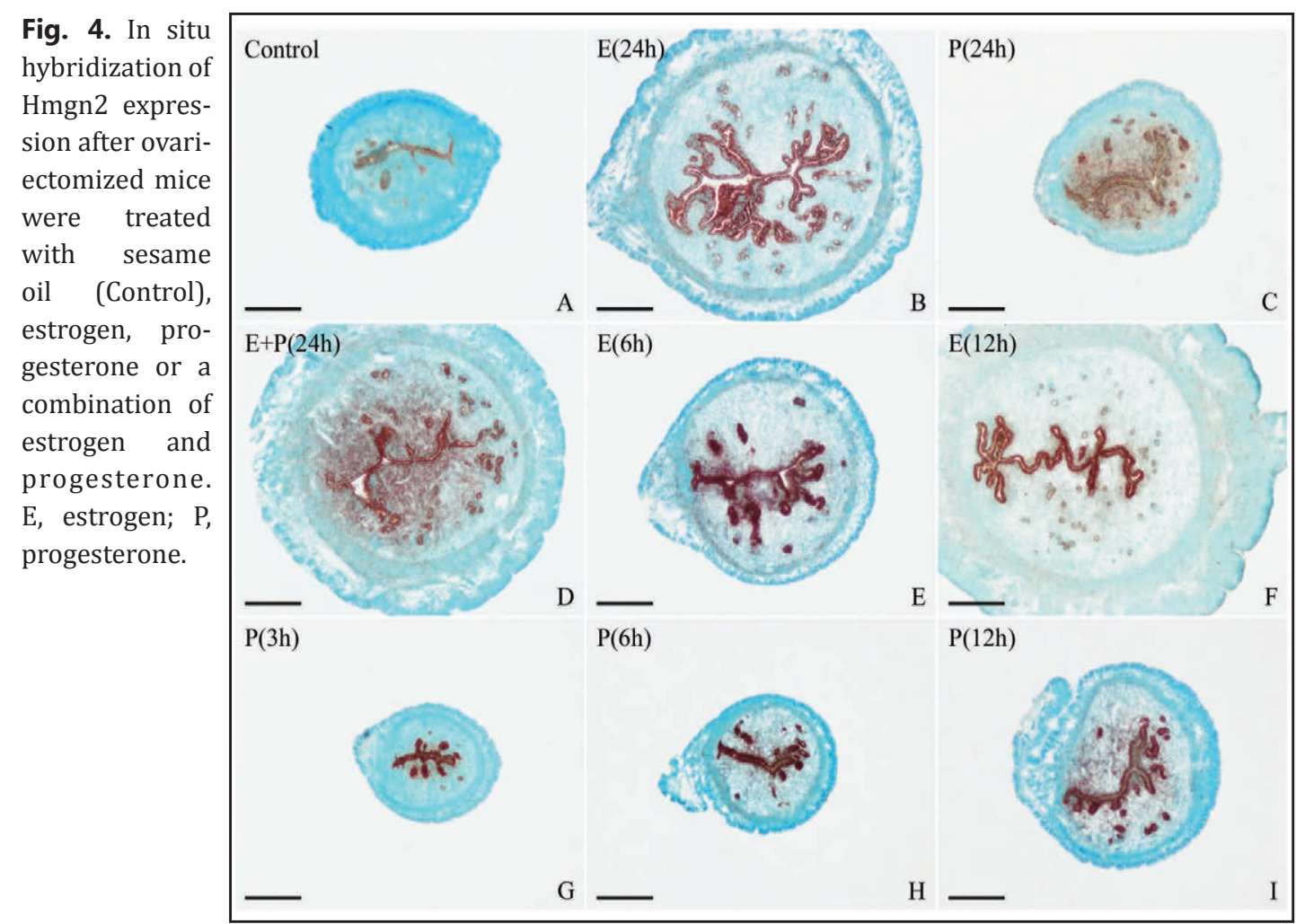

injection, then enhanced at $6 \mathrm{~h}$ and remained more or less steady through 12 and $24 \mathrm{~h}$ (Fig. 4B, E and F). By real-time PCR, the level of Hmgn2 mRNA showed a marked increase at $3 \mathrm{~h}$, peaking at $6 \mathrm{~h}$, followed by a decline and reached a nadir at 24, although estrogen could promote the expression of Hmgn2 in the uteri of ovariectomized mice (Fig. 5B). To further elucidate estrogen regulation of Hmgn2 expression, uterine epithelial cells were treated with estrogen in vitro. Addition of estrogen resulted in elevated mRNA level for Hmgn2, but the up-regulation was abrogated by estrogen receptor (ER) antagonist ICI 182, 780 (Fig. 5C). Consistently, a heightened expression was noted in the uterine luminal and glandular epithelium between 3 to 24 h post-injection of progesterone (Fig. 4C, G-I). In the meantime, Hmgn2 mRNA signal was also accumulated in the stromal cells at 12 and $24 \mathrm{~h}$ after ovariectomized mice were treated with progesterone (Fig. 4C and I). Further analyses by real-time PCR revealed an increased expression in uterine Hmgn2 mRNA, but the rise exhibited a time-dependent reduction (Fig. 5D). In the in vitro cultured uterine stromal cells, progesterone augmented the expression of Hmgn2, which reached a maximum at $24 \mathrm{~h}$, whereas progesterone receptor (PR) antagonist RU486 eliminated the up-regulation elicited by progesterone (Fig. 5E and F).

\section{Effects of Hmgn2 on the expression of Cdh1, Cdh2, Ctnnb1 and Muc1 in uterine epithelial cells}

Since Hmgn2 mRNA signal was visualized in uterine luminal epithelium on day 4 (receptive phase) of pregnancy, we examined the regulation of Hmgn2 on the expression of cadherin 1 (Cdh1, also known as E-cadherin), Cdh2 (also referred to as N-cadherin), catenin (cadherin associated protein), beta 1 (Ctnnb1, also known as $\beta$-catenin), mucin 1, transmembrane (Muc1) which were important adhesion molecules during embryo implantation [17-20]. Treatment with siRNA targeted to Hmgn2, which efficiently suppressed the level of Hmgn2 mRNA, led to a remarkable reduction in the expression of Cdh1, Cdh2 and Ctnnb1, while the level of Muc1 mRNA exhibited a dramatic increase (Fig. 6A). In contrast, constitutive activation of Hmgn2 apparently raised the expression of Hmgn2, Cdh1, Cdh2 and Ctnnb1 in uterine epithelial cells and repressed the expression of Muc1 (Fig. 6B and C). 

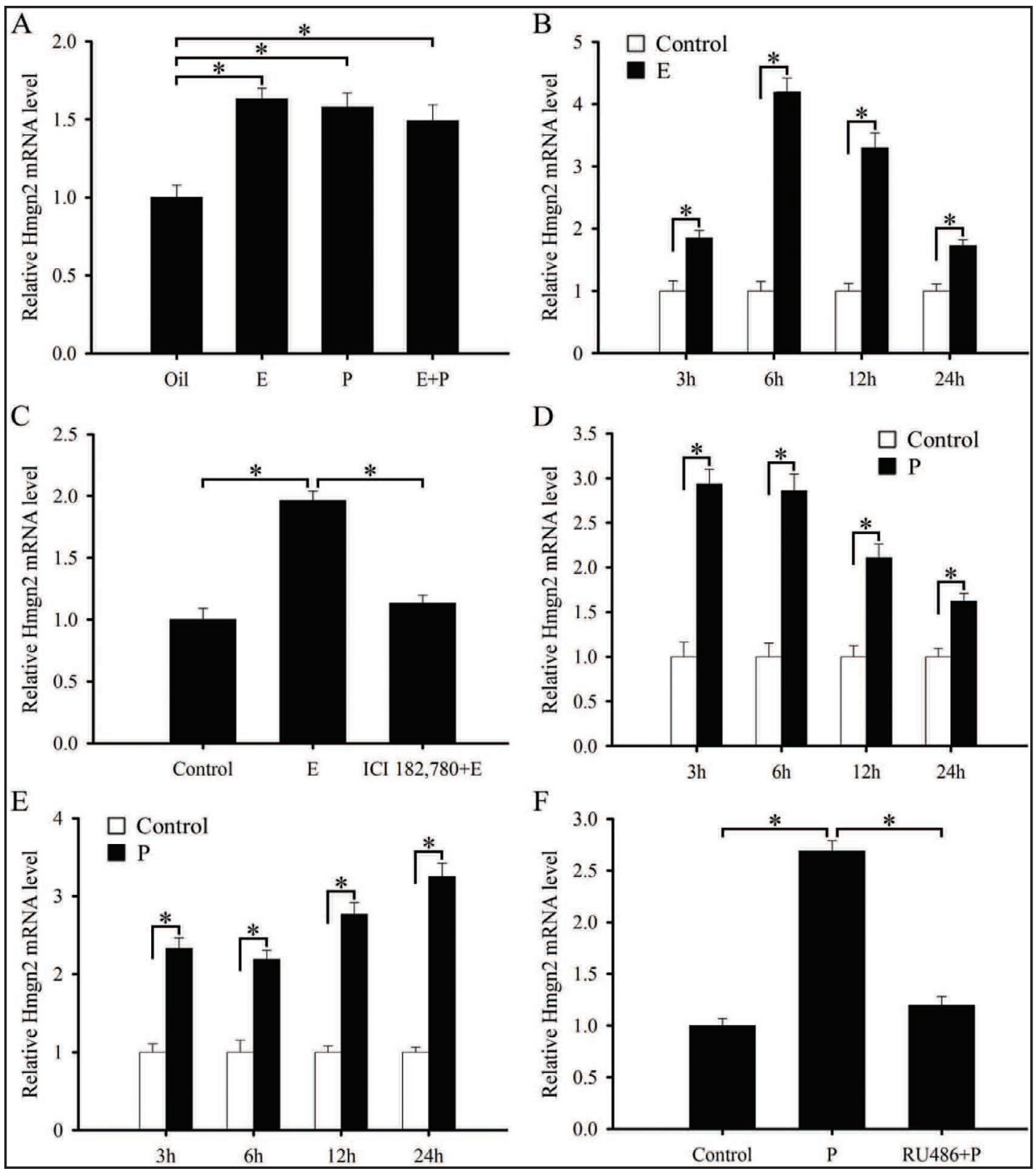

Fig. 5. Hormonal regulation of Hmgn2 expression. A, Real-time PCR analysis of Hmgn2 expression after ovariectomized mice were treated with sesame oil, estrogen, progesterone or a combination of estrogen and progesterone for $24 \mathrm{~h}$. B, Real-time PCR analysis of Hmgn2 expression in ovariectomized mouse uteri after injection of estrogen for 3, 6, 12 and $24 \mathrm{~h}$. C, Real-time PCR analysis of Hmgn2 expression after uterine epithelial cells were treated with estrogen or both estrogen and ICI 182,780. D, Real-time PCR analysis of Hmgn2 expression in ovariectomized mouse uteri after injection of progesterone for 3, 6, 12 and $24 \mathrm{~h}$. E, Real-time PCR analysis of Hmgn2 expression in uterine stromal cells treated with progesterone for 3, 6, 12 and $24 \mathrm{~h}$. F, Real-time PCR analysis of Hmgn2 expression after uterine stromal cells were treated with progesterone or both progesterone and RU486.

Hmgn2 mediates the effects of estrogen on the expression of Cdh1, Cdh2 and Ctnnb1 in uterine epithelial cells

It is generally accepted that estrogen is of great importance to embryo implantation in mice $[1,2,21]$. Moreover, previous studies have found that Cdh1, Cdh2 and Ctnnb1 were the established estrogen-modulated genes [22-26]. In the in vitro cultured uterine epithelial cells, 


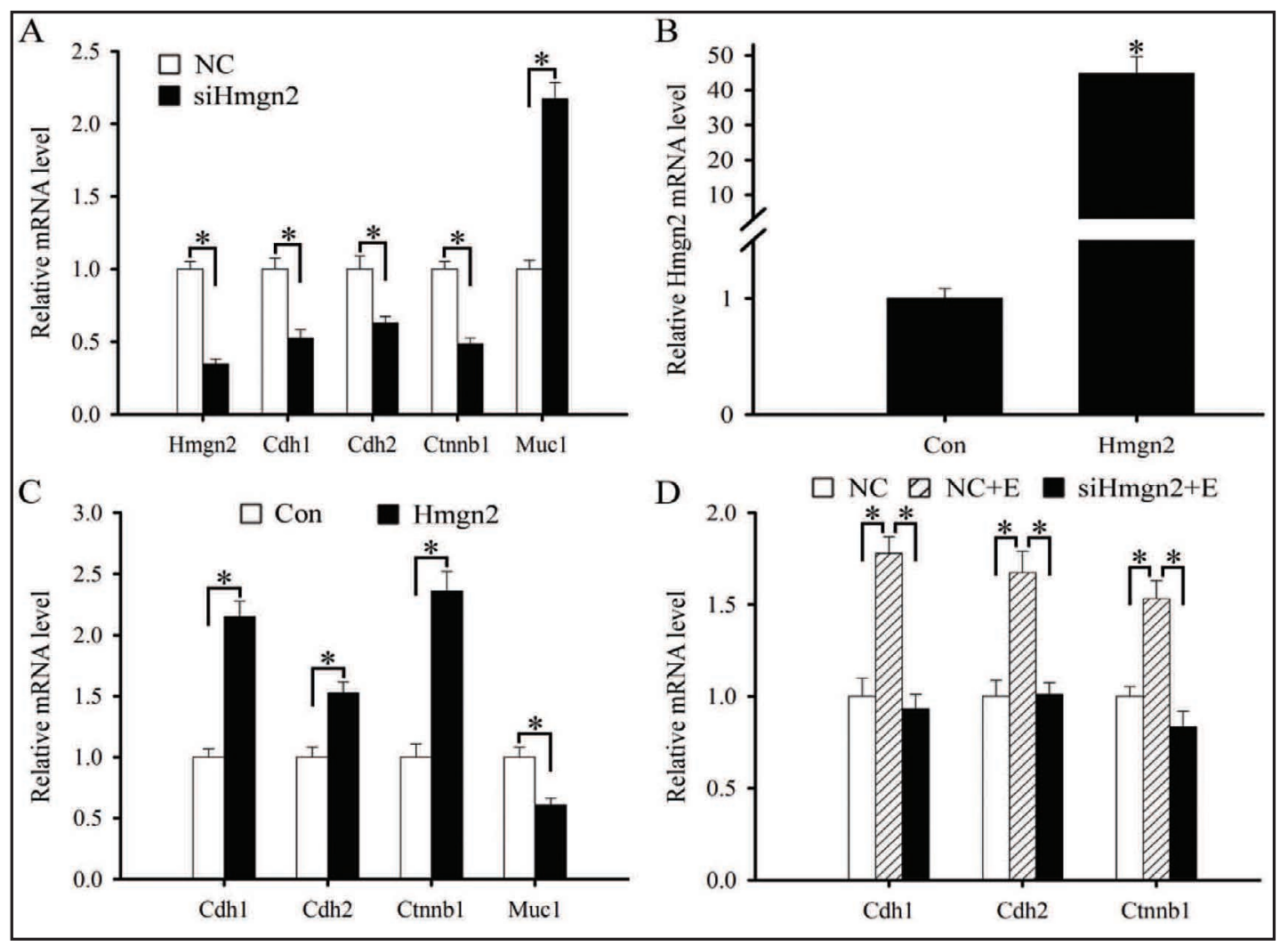

Fig. 6. Hmgn 2 mediates the effects of estrogen on the expression of Cdh1, Cdh2 and Ctnnb1 in uterine epithelial cells. A, Effects of Hmgn2 siRNA on the expression of Hmgn2, Cdh1, Cdh2, Ctnnb1 and Muc1. B, Hmgn2 expression after uterine epithelial cells were transfected with Hmgn2 overexpression plasmid. C, Effects of Hmgn2 overexpression on the expression of Cdh1, Cdh2, Ctnnb1 and Muc1. D, Hmgn2 mediated the effects of estrogen on the expression of Cdh1, Cdh2 and Ctnnb1. After transfection with Hmgn2 siRNA and addition of estrogen, the expression of $\mathrm{Cdh} 1, \mathrm{Cdh} 2$ and Ctnnb1 was determined by real-time PCR. NC, negative control; siHmgn2, Hmgn2 siRNA; Con, empty pcDNA3.1 vector; Hmgn2, Hmgn2 overexpression plasmid.

estrogen could also stimulate the expression of Cdh1, Cdh2 and Ctnnb1 (Fig. 6D). As stated above, estrogen could induce the accumulation of Hmgn2 which governed the expression of Cdh1, Cdh2 and Ctnnb1. We next sought to ascertain whether Hmgn2 might mediate the regulation of estrogen on the expression of Cdh1, Cdh2 and Ctnnb1. To address this, we administrated estrogen to epithelial cells treated with Hmgn 2 siRNA and then monitored the expression of Cdh1, Cdh2 and Ctnnb1. The result found that silencing of Hmgn2 abolished the up-regulation of Cdh1, Cdh2 and Ctnnb1 elicited by estrogen (Fig. 6D).

\section{Effects of Hmgn2 on the proliferation and differentiation of uterine stromal cells}

Decidualization involves extensive proliferation and differentiation of uterine stromal cells [2]. As stromal cell proliferation is the first step of decidualization, we first tested the effects of Hmgn2 on stromal cell proliferation. After transfection with Hmgn2 siRNA or overexpression plasmid, the proliferation activity of uterine stromal cells did not show any obvious change (Fig. 7A and B). To unveil the role of Hmgn2 in the differentiation of uterine stromal cells, we next analyzed its effects on the expression of prolactin family 8 , subfamily a, member 2 (Prl8a2) and prolactin family 3, subfamily c, member 1 (Prl3c1), two wellknown markers for uterine stromal differentiation during decidualization [15]. The result demonstrated that knockdown of Hmgn2 could down-regulate the expression of Prl8a2 and Prl3c1, whereas overexpression of Hmgn2 exhibited the opposite effects (Fig. 7C and D). 


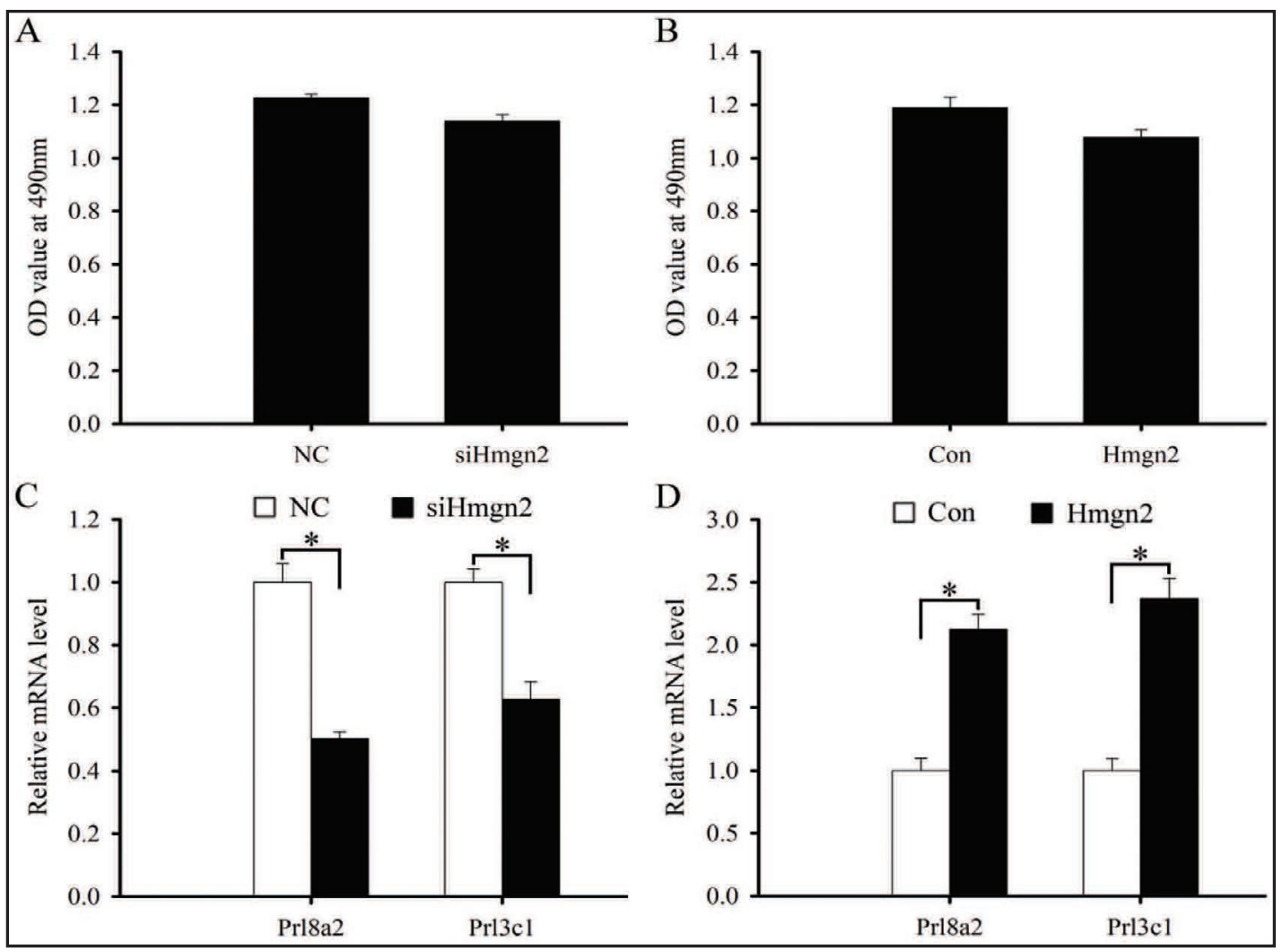

Fig. 7. Effects of Hmgn 2 on the proliferation and differentiation of uterine stromal cells. A, Effects of Hmgn2 siRNA on the proliferation of uterine stromal cells. B, Effects of Hmgn2 overexpression on the proliferation of uterine stromal cells. C, Effects of Hmgn2 siRNA on the expression of Prl8a2 and Prl3c1. D, Effects of Hmgn2 overexpression on the expression of Prl8a2 and Prl3c1.

Hmgn2 mediates the effects of progesterone on the differentiation of uterine stromal cells

It has previously been reported that progesterone was essential for the differentiation of uterine stromal cells during decidualization $[2,27,28]$. As described above, progesterone could promote the expression of Hmgn2 which played an important role in stromal differentiation. We next asked whether Hmgn2 might mediate the effects of progesterone on the differentiation of uterine stromal cells. After transfection with Hmgn2 siRNA, the induction of Prl8a2 and Prl3c1 by progesterone was evidently weakened (Fig. 8A and B).

Hmgn2 mediates the regulation of progesterone on Hand2 expression in uterine stromal cells

Because Hmgn2 was involved in transcriptional regulation of numerous genes [11, $14,29]$, we analyzed its influence on the expression of decidualization-related gene heart and neural crest derivatives expressed transcript 2 (Hand2), whose expression in the decidua overlapped with that of Hmgn2 [30]. After transfection with Hmgn2 siRNA, uterine stromal cells revealed a notable reduction in the level of Hand2 mRNA (Fig. 8C). Conversely, constitutive activation of Hmgn2 resulted in an obvious enhancement in the expression of Hand2 (Fig. 8C). Previous evidence had displayed that Hand2 was a well-characterized progesterone-responsive gene [28, 31]. We next dissected the role of Hmgn2 in progesterone regulation of Hand2. In uterine stromal cells, progesterone could dramatically boost the expression of Hand2. However, once Hmgn2 siRNA was introduced into uterine stromal cells, the stimulation of progesterone on Hand2 expression was efficiently abrogated (Fig. 8D). 


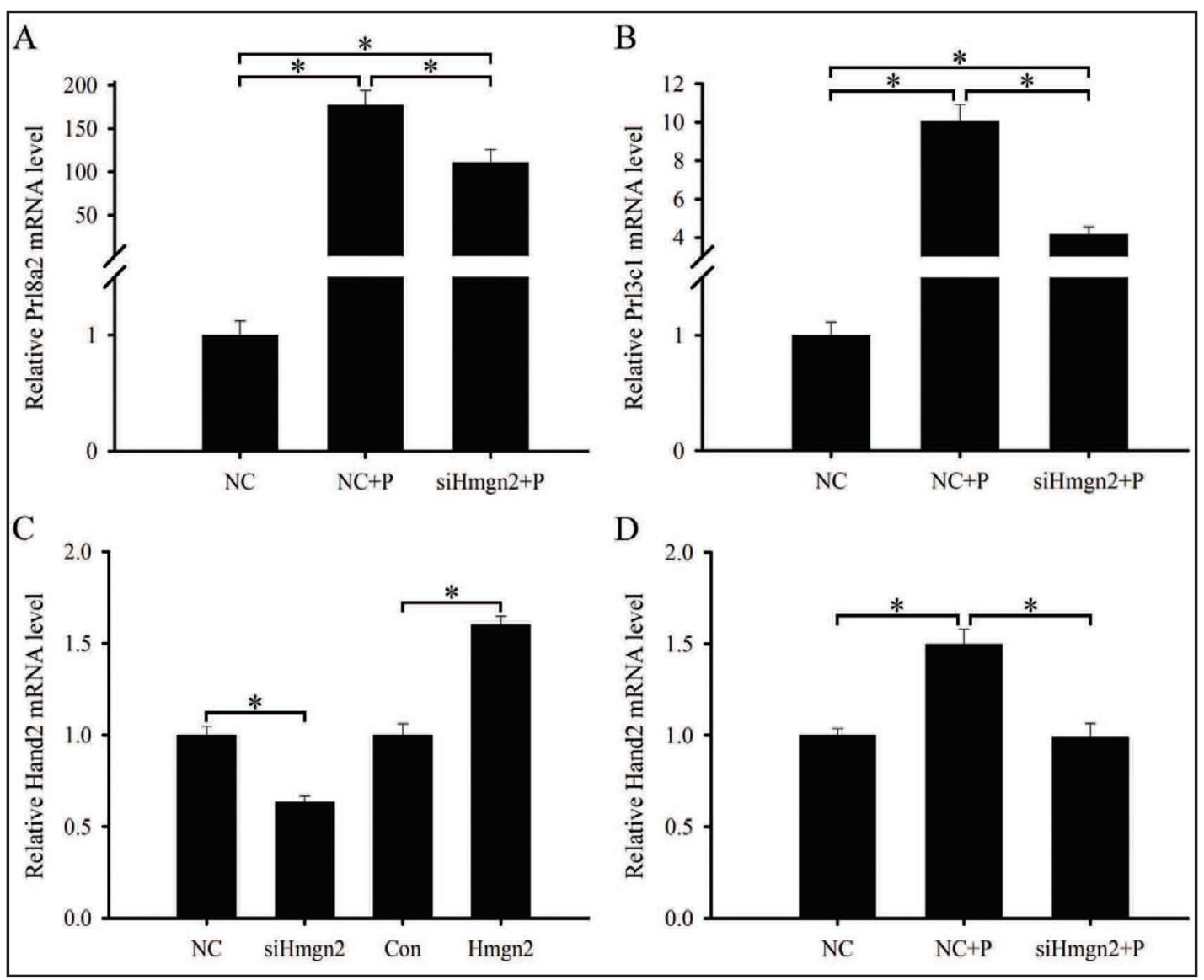

Fig. 8. Hmgn2 mediates the effects of progesterone on stromal differentiation and Hand 2 expression. A and B, Hmgn2 mediated the effects of progesterone on the expression of Prl8a2 and Prl3c1. After transfection with Hmgn 2 siRNA and addition of progesterone, the expression of Prl8a2 and Prl3c1 was determined by real-time PCR. C, Effects of Hmgn2 siRNA or overexpression on Hand2 expression. D, Hmgn2 mediated the effects of progesterone on Hand2 expression.

\section{Discussion}

Successful implantation requires the development of embryo to implantation-competent blastocyst and the synchronized transformation of uteri into a receptive stage [1-3]. Previous study has confirmed that Hmgn 2 could play an important role in preimplantation embryonic development [12], but its biological function during embryo implantation remains unknown. In this study, we found that on day 4 of pregnancy when the uterus is receptive, Hmgn 2 mRNA was visualized in the luminal epithelium, which was consistent with that in the delayed uterus, suggesting a potential role of Hmgn2 in uterine receptivity. It is well known that reciprocal interaction between the activated blastocyst and receptive uterus may trigger the process of implantation, which is classified into three stages: apposition, attachment and penetration, coinciding with the elevated expression of Hmgn2 mRNA at implantation sites on day 5 when blastocyst implantation is in progress and attachment reaction becomes more prominent $[1,20,32]$. In uterine epithelium cells, Hmgn 2 could stimulate the expression of Cdh1 and Cdh2 that were the transmembrane adhesion molecules and might mediate the adhesion of uterine epithelium and blastocyst trophoblast during implantation $[17,18]$. Uterine-specific deletion of Cdh1 resulted in the failure of implantation due to a lack of epithelial adhesion junction [17]. Further analysis evidenced that ablation of $\operatorname{Cdh} 1$ in uterine epithelium led to a loss of Ctnnb1 which might participate in the formation of the epithelial adhesion junction in cooperation with Cdh1 $[17,19,20]$. Moreover, deficiency of Ctnnb1 resulted in 
the impairment of epithelial junction [19]. In uterine epithelial cells, Hmgn2 also induced the accumulation of Ctnnb1 mRNA. Together these observations indicate that Hmgn2 may play a crucial role in attachment reaction during embryo implantation. This notion was further reinforced by the finding that Hmgn2 repressed the expression of Muc1 which might act as an antiadhesive molecule to prevent the attachment of blastocyst to uterine epithelium [20, 33]. In addition, Hmgn 2 mRNA signal was distinctly observed in the embryo on days 6-8 of pregnancy, implying that Hmgn2 may be implicated in regulating embryonic development. Indeed, ablation of Hmgn2 in mice resulted in embryonic lethality [9, 11].

Estrogen is crucial for blastocyst implantation into a progesterone-primed receptive uterus in rodent and its action is primarily mediated through activation of the intracellular ER $[1,2]$. The present study showed that estrogen could up-regulate the expression of Hmgn2 in uterine epithelia in vivo and in vitro. Moreover, the up-regulation was abrogated by ER antagonist ICI 182, 780, suggesting ER requirement for this induction. It has previously reported that Cdh1, Cdh2 and Ctnnb1 were the established estrogen-targeting genes [22-26]. This was consistent with our observation in uterine epithelial cells. Moreover, knockdown of Hmgn2 efficiently eliminated the regulation of estrogen on the expression of Cdh1, Cdh2 and Ctnnb1, indicating that Hmgn2 is a critical mediator of estrogen in regulating epithelial function.

Accompanying with the onset of embryo implantation, uterine stromal cells undergo extensive proliferation and then differentiation into decidual cells [1,2]. With the progression of decidualization, Hmgn2 mRNA signal was visibly noted in the decidua. In the meantime, Hmgn2 might significantly promote the expression of Prl8a2 and Prl3c1 which were two wellestablished stromal differentiation markers during decidualization, but did not affect the proliferation of uterine stromal cells. Taken together, these results demonstrate that Hmgn2 plays an important role in the differentiation of stromal cells during uterine decidualization. Meanwhile, knockdown of Hmgn5, which was a novel discovered member of Hmgn family and crucial for decidualization [15], enhanced the expression of Hmgn2 in uterine stromal cells (our unpublished data), suggesting that Hmgn2 may functionally compensate the role of Hmgn5 in stromal differentiation. Further analysis revealed that Hmgn2 could stimulate the expression of Hand 2 whose knockdown damaged the stromal differentiation in both mice and human [30,34], implying that Hand2 may be a downstream regulator of Hmgn2 during decidualization. Previous studies have established that Hmgn2 was involved in transcriptional regulation of numerous genes by affecting histone modifications and activity of ATP-dependent chromatin remodeling complexes, or binding to chromatin regulatory sites such as Dnase I-hypersensitive sites, enhancers and promoters [11, 14, 29, 35], but the regulatory mechanism of Hmgn2 on the expression of Hand2 in uterine stromal cells remains to be determined.

It has long been recognized that ovarian progesterone signaling via PR is essential for decidualization $[2,36]$. In uterine stromal cells, progesterone could boost the expression of Hmgn2, but the increase was eliminated by PR antagonist RU486. Simultaneously, silencing of Hmgn2 by specific siRNA significantly impeded the induction of progesterone on the differentiation of uterine stromal cells as evidenced by the reduced expression of differentiation markers Prl8a2 and Prl3c1. Collectively, these results reveal that Hmgn2 is a critical downstream target of progesterone in regulating stromal differentiation during decidualization. Previous studies have found that progesterone induced the accumulation of Hand2 mRNA in uterine stromal cells $[28,31]$. However, once transfection with Hmgn2 siRNA, the induction of Hand2 by progesterone was abolished, implying that Hmgn2 is required in the crosstalk between progesterone and Hand2.

\section{Conclusion}

Hmgn2 may play an important role in embryo implantation and decidualization and act downstream of progesterone to regulate the differentiation of uterine stromal cells through targeting Hand2. 


\section{Cellular Physiology Cell Physiol Biochem 2017;44:1681-1695 \begin{tabular}{ll|l} 
and Biochemistry & $\begin{array}{l}\text { DOI: 10.1159/000485775 } \\
\text { Published OnIIne: December 06, } 2017\end{array}$ & $\begin{array}{l}\text { (c) } 2017 \text { The Author(s). Published by S. Karger AG, Basel } \\
\text { www.karger.com/cpb }\end{array}$ \\
\hline
\end{tabular}}

Li et al.: Hmgn2 Regulation in Mouse Uterus

\section{Acknowledgements}

This work was financially supported by National Natural Science Foundation of China (31472158 and 31672503), National Key Research and Development Program of China, Stem Cell and Translational Research (2017YFA0105101) and Special Funds for Scientific Research on Public Causes (201303119).

\section{Disclosure Statement}

The authors declare that they have no Disclosure Statement.

\section{References}

1 Cha J, Sun X, Dey SK: Mechanisms of implantation: strategies for successful pregnancy. Nat Med 2012;18:1754-1767.

-2 Zhang S, Lin H, Kong S, Wang S, Wang H, Wang H, Armant DR: Physiological and molecular determinants of embryo implantation. Mol Aspects Med 2013;34:939-980.

- 3 Ran H, Kong S, Zhang S, Cheng J, Zhou C, He B, Xin Q, Lydon JP, DeMayo FJ, Feng GS, Xia G, Lu Z, Wang C, Wang H: Nuclear Shp2 directs normal embryo implantation via facilitating the ERalpha tyrosine phosphorylation by the Src kinase. Proc Natl Acad Sci U S A 2017;114:4816-4821.

-4 Salker MS, Steel JH, Hosseinzadeh Z, Nautiyal J, Webster Z, Singh Y, Brucker S, Lang F, Brosens JJ: Activation of SGK1 in Endometrial Epithelial Cells in Response to PI3K/AKT Inhibition Impairs Embryo Implantation. Cell Physiol Biochem 2016;39:2077-2087.

-5 Wang H, Dey SK: Roadmap to embryo implantation: clues from mouse models. Nat Rev Genet 2006;7:185199.

-6 Cui N, Wang H, Wang W, Zhang J, Xu Y, Jiang L, Yang A, Hao G: Impact of Body Mass Index on Outcomes of In vitro Fertilization/Intracytoplasmic Sperm Injection Among Polycystic Ovarian Syndrome Patients. Cell Physiol Biochem 2016;39:1723-1734.

7 Liao Y, Jiang Y, He H, Ni H, Tu Z, Zhang S, Wang B, Lou J, Quan S, Wang H: NEDD8-mediated neddylation is required for human endometrial stromal proliferation and decidualization. Hum Reprod 2015;30:16651676.

8 Tamura I, Ohkawa Y, Sato T, Suyama M, Jozaki K, Okada M, Lee L, Maekawa R, Asada H, Sato S, Yamagata Y, Tamura H, Sugino N: Genome-wide analysis of histone modifications in human endometrial stromal cells. Mol Endocrinol 2014;28:1656-1669.

-9 Furusawa T, Cherukuri S: Developmental function of HMGN proteins. Biochim Biophys Acta 2010;1799:6973.

$>10$ Gonzalez-Romero R, Eirin-Lopez JM, Ausio J: Evolution of high mobility group nucleosome-binding proteins and its implications for vertebrate chromatin specialization. Mol Biol Evol 2015;32:121-131.

-11 Rochman M, Taher L, Kurahashi T, Cherukuri S, Uversky VN, Landsman D, Ovcharenko I, Bustin M: Effects of HMGN variants on the cellular transcription profile. Nucleic Acids Res 2011;39:4076-4087.

12 Mohamed OA, Bustin M, Clarke HJ: High-mobility group proteins 14 and 17 maintain the timing of early embryonic development in the mouse. Dev Biol 2001;229:237-249.

$>13$ Kulkeaw K, Inoue T, Mizuochi C, Horio Y, Ishihama Y, Sugiyama D: Ectopic expression of Hmgn2 antagonizes mouse erythroid differentiation in vitro. Cell Biol Int 2012;36:195-202.

14 Nagao M, Lanjakornsiripan D, Itoh Y, Kishi Y, Ogata T, Gotoh Y: High mobility group nucleosome-binding family proteins promote astrocyte differentiation of neural precursor cells. Stem Cells 2014;32:2983-2997.

15 Li DD, Zhao SY, Yang ZQ, Duan CC, Guo CH, Zhang HL, Geng S, Yue ZP, Guo B: Hmgn5 functions downstream of Hoxa10 to regulate uterine decidualization in mice. Cell Cycle 2016;15:2792-2805.

-16 Li F, Devi YS, Bao L, Mao J, Gibori G: Involvement of cyclin D3, CDKN1A (p21), and BIRC5 (Survivin) in interleukin 11 stimulation of decidualization in mice. Biol Reprod 2008;78:127-133. 


\section{Cellular Physiology Cell Physiol Biochem 2017;44:1681-1695 \begin{tabular}{ll|l} 
DOI: 10.1159/000485775 & $\begin{array}{l}\text { O 2017 The Author(s). Published by S. Karger AG, Basel } \\
\text { www.karger.com/cpb }\end{array}$ \\
\hline and Biochemistry Published online: December 06, 2017
\end{tabular}}

Li et al.: Hmgn2 Regulation in Mouse Uterus

17 Reardon SN, King ML, MacLean JA, 2nd, Mann JL, DeMayo FJ, Lydon JP, Hayashi K: CDH1 is essential for endometrial differentiation, gland development, and adult function in the mouse uterus. Biol Reprod 2012;86:141, 141-110.

18 Singh H, Aplin JD: Adhesion molecules in endometrial epithelium: tissue integrity and embryo implantation. J Anat 2009;215:3-13.

19 Jeong JW, Lee HS, Franco HL, Broaddus RR, Taketo MM, Tsai SY, Lydon JP, DeMayo FJ: beta-catenin mediates glandular formation and dysregulation of beta-catenin induces hyperplasia formation in the murine uterus. Oncogene 2009;28:31-40.

20 Dey SK, Lim H, Das SK, Reese J, Paria BC, Daikoku T, Wang H: Molecular cues to implantation. Endocr Rev 2004;25:341-373.

-21 Ma WG, Song H, Das SK, Paria BC, Dey SK: Estrogen is a critical determinant that specifies the duration of the window of uterine receptivity for implantation. Proc Natl Acad Sci U S A 2003;100:2963-2968.

22 Gu X, Yu JJ, Ilter D, Blenis N, Henske EP, Blenis J: Integration of mTOR and estrogen-ERK2 signaling in lymphangioleiomyomatosis pathogenesis. Proc Natl Acad Sci U S A 2013;110:14960-14965.

23 Hou X, Tan Y, Li M, Dey SK, Das SK: Canonical Wnt signaling is critical to estrogen-mediated uterine growth. Mol Endocrinol 2004;18:3035-3049.

24 Chen YJ, Li HY, Huang CH, Twu NF, Yen MS, Wang PH, Chou TY, Liu YN, Chao KC, Yang MH: Oestrogeninduced epithelial-mesenchymal transition of endometrial epithelial cells contributes to the development of adenomyosis. J Pathol 2010;222:261-270.

25 Wada-Hiraike O, Hiraike H, Okinaga H, Imamov O, Barros RP, Morani A, Omoto Y, Warner M, Gustafsson JA: Role of estrogen receptor beta in uterine stroma and epithelium: Insights from estrogen receptor beta-/mice. Proc Natl Acad Sci U S A 2006;103:18350-18355.

-26 Laviolette LA, Hodgkinson KM, Minhas N, Perez-Iratxeta C, Vanderhyden BC: 17beta-estradiol upregulates GREB1 and accelerates ovarian tumor progression in vivo. Int J Cancer 2014;135:1072-1084.

-27 Li Q Kannan A, Wang W, Demayo FJ, Taylor RN, Bagchi MK, Bagchi IC: Bone morphogenetic protein 2 functions via a conserved signaling pathway involving Wnt4 to regulate uterine decidualization in the mouse and the human. J Biol Chem 2007;282:31725-31732.

-28 Yu HF, Tao R, Yang ZQ, Wang K, Yue ZP, Guo B: Ptn functions downstream of C/EBPbeta to mediate the effects of cAMP on uterine stromal cell differentiation through targeting Hand2 in response to progesterone. J Cell Physiol 2017 DOI: 10.1002/jcp.26067.

29 Deng T, Zhu ZI, Zhang S, Postnikov Y, Huang D, Horsch M, Furusawa T, Beckers J, Rozman J, Klingenspor M, Amarie O, Graw J, Rathkolb B, Wolf E, Adler T, Busch DH, Gailus-Durner V, Fuchs H, Hrabe de Angelis M, van der Velde A, Tessarollo L, Ovcherenko I, Landsman D, Bustin M: Functional compensation among HMGN variants modulates the DNase I hypersensitive sites at enhancers. Genome Res 2015;25:1295-1308.

30 Huyen DV, Bany BM: Evidence for a conserved function of heart and neural crest derivatives expressed transcript 2 in mouse and human decidualization. Reproduction 2011;142:353-368.

-31 Li Q Kannan A, DeMayo FJ, Lydon JP, Cooke PS, Yamagishi H, Srivastava D, Bagchi MK, Bagchi IC: The antiproliferative action of progesterone in uterine epithelium is mediated by Hand2 Science 2011;331:912916.

-32 Daikoku T, Cha J, Sun X, Tranguch S, Xie H, Fujita T, Hirota Y, Lydon J, DeMayo F, Maxson R, Dey SK: Conditional deletion of Msx homeobox genes in the uterus inhibits blastocyst implantation by altering uterine receptivity. Dev Cell 2011;21:1014-1025.

-33 Nallasamy S, Li Q, Bagchi MK, Bagchi IC: Msx homeobox genes critically regulate embryo implantation by controlling paracrine signaling between uterine stroma and epithelium. PLoS Genet 2012;8:e1002500.

34 Yu HF, Yue ZP, Wang K, Yang ZQ, Zhang HL, Geng S, Guo B: Gja1 acts downstream of Acvr1 to regulate uterine decidualization via Hand2 in mice. J Endocrinol 2017;233:145-157.

35 Zhu N, Hansen U: Transcriptional regulation by HMGN proteins. Biochim Biophys Acta 2010;1799:74-79.

-36 Large MJ, DeMayo FJ: The regulation of embryo implantation and endometrial decidualization by progesterone receptor signaling. Mol Cell Endocrinol 2012;358:155-165. 